\title{
EL TRABAJO COMO MEDIO DE SANTIFICACIÓN DE LA FAMILIA
}

\author{
WORK AS A FAMILY SANCTIFICATION MEANS
}

P. Carlos Rosell De Almeida

\section{RESUMEN}

El presente artículo está estructurado en dos partes. En la primera, hacemos una síntesis sobre la teología del trabajo, para ello es necesario recurrir a la Sagrada Escritura, los Padres de la Iglesia y el Magisterio. Hemos querido desarrollar primero este tema dado que nuestra intención es mostrar qué nos dice la fe sobre el trabajo humano. En la segunda parte, señalaremos la importancia del trabajo como medio de santificación de la familia.

\section{Palabras clave}

Trabajo, familia, bien común, educación, responsabilidad.

\section{ABTRACT}

This work is divided into two parts. In the first one, we will make a summary about the theology of work, and for this it is necessary to resort to the Holy Scripture, the Church Fathers and the Magisterium. We intend to develop this subject first since our intention is to show what our faith tells us about human work. In the second part, we will explain the importance of working as a family sanctification means.

\section{Keywords}

Work, family, common well-being, education, responsibility

\section{INTRODUCCIÓN}

La cuestión del trabajo, qué duda cabe, es un tema que puede ser abordado desde diversas perspectivas. Cada una de ellas, trae visiones legítimas, y con luces valiosas para entender la verdad más profunda sobre el trabajo humano. En este sentido, nuestra intención es exponer algunas pinceladas desde la Doctrina de la Iglesia sobre la relación entre el trabajo y la familia. Pretendemos, al respecto, resaltar que esta actividad humana tan esencial para el desarrollo de la persona humana también tiene repercusiones en el despliegue de la vida cristiana en una familia. Por decirlo de otra manera, el trabajo no es indiferente al llamado a la santidad que atañe a todo bautizado.

\section{TEOLOGÍA DEL TRABAJO}

\section{El trabajo en la Sagrada Escritura}

Una lectura atenta de la Sagrada Escritura permite afirmar que el trabajo no es un castigo

Ingeniero Civil titulado (1993) y egresado de la Universidad Nacional de Ingeniería. Fue ordenado sacerdote para la Arquidiócesis de Lima el año 2004. Obtuvo el grado de Doctor en Sagrada Teología por la Universidad de Navarra (España) con la tesis Escatología ortodoxa que fue premio extraordinario (2007). Desde el año 2010 es Rector del Seminario Conciliar de Santo Toribio de Mogrovejo (Lima). Además se desempeña como profesor de Teología en la Facultad de Teología Pontificia y Civil de Lima dictando fundamentalmente las asignaturas de Dios Uno y Trino y escatología. 
impuesto por Dios al hombre tras la caída original. Al contrario, el trabajo está presente antes del pecado de Adán y Eva. En efecto, Dios le da la orden de dominar la tierra (cf. Gn 1, 28). De esa manera, el hombre aparece como un ser que está destinado a trabajar. Trabajando se perfecciona como persona. Lo que sí trae el pecado es hacer arduo y fatigoso el trabajo (cf. Gn 3, 17)

En el Nuevo Testamento encontramos algunas enseñanzas esenciales sobre el trabajo. En primer lugar, debemos afirmar que Jesús mismo fue trabajador. Es llamado el carpintero o el artesano (cf. Mc 6, 3). Dios encarnado trabajó con sus propias manos. ${ }^{2}$

Además, Jesús llama para que lo sigan a personas trabajadoras. Los Apóstoles tenían sus ocupaciones, unos eran pescadores, pensemos en Andrés, Pedro, Santiago y Juan (cf. Mc 1, 16-19). Otro, como es el caso de Mateo o Levi, era recaudador de impuestos (cf. Mt 9, 9). Es decir que el Señor se fija en quienes estaban ocupados.

La Iglesia naciente es consciente de que el trabajo es un medio de subsistencia. El mismo San Pablo afirma que quiere trabajar para no ser gravoso (cf. Hch 18, 3; 1 Co 4, 12). $\mathrm{Y}$ a los Tesalonicenses les remarca que si no quieren trabajar que no coman (cf. 2 Ts 3,10 ). El contexto en que les da ese imperativo es que algunos de la comunidad de Tesalónica pensaban que la segunda venida del Señor sería inminente y por esa razón habían dejado de trabajar.

Hagamos referencia a tres pasajes que podemos relacionarlos con la santificación del trabajo. En $\mathrm{Rm} 12,1$ se indica que estamos llamados a ser hostias vivas, santas agradables a Dios. Esto lo podemos aplicar al cristiano que trabajando se ofrece a Dios. En 1 P 2,5, el apóstol San Pedro afirma que los cristianos son piedras vivas de un edificio espiritual y gracias al sacerdocio santo que tienen pueden ofrecer sacrificios espirituales a Dios por medio de Cristo. Este texto expresa lo propio del sacerdocio común. Un tercer pasaje es 1 Co 10,31, ahí San Pablo afirma: "Ya comáis, ya bebáis, o hagáis cualquier otra cosa, hacedlo todo para gloria de Dios". Podríamos afirmar, sin alterar el sentido del texto: ya trabajéis hacedlo para gloria de Dios.

\section{El trabajo en la Tradición viva de la Iglesia}

Quisiera referirme, aunque sea solo de una manera muy breve, a lo que nos refieren los testimonios de la Tradición de la Iglesia. En primer lugar, convendría citar un documento de inicios del siglo II, llamado la Didaje. En él conocemos cómo era la vida de la Iglesia primitiva. Una de las recomendaciones que se indica es que en la comunidad cristiana todos deben emplearse en algo

Todo el que llegue a vosotros en nombre del Señor, sea recibido; luego examinándole, le conoceréis... Si el que llega es un caminante, no permanecerá entre vosotros más de dos días $o$, si hubiera necesidad, tres. Pero si quiere establecerse entre vosotros, teniendo un oficio, que trabaje y así se alimente. Más sino tiene oficio, proveed según vuestra prudencia, de modo que no viva entre nosotros ningún cristiano ocioso. Si no quiere hacerlo así, es un traficante de Cristo; estad alerta contra tales. (Didaje 12)

Encontramos dentro de las enseñanzas de los Padres de la Iglesia la idea de que el trabajo digno es un medio eficaz para vivir en comunión con Dios. No solo la oración -que por cierto debe ser siempre lo primero- sino las ocupaciones habituales son una vía para estar unidos al Señor. A este respecto, quisiera citar unas hermosas palabras de San Juan Crisóstomo (†407):

\footnotetext{
"El Hijo de Dios con su encarnación se ha unido, en cierto modo, con todo hombre. Trabajó con manos de hombre, pensó con inteligencia de hombre, obró con voluntad de hombre, amó con corazón de hombre. Nacido de la Virgen María, se hizo verdaderamente uno de los nuestros, semejante en todo a nosotros, excepto en el pecado". CONCILIO VATICANO II, Constitución pastoral Gaudium et spes, n. 22.
} 
Una mujer ocupada en la cocina o en coser una tela puede siempre elevar su pensamiento al cielo e invocar al Señor con fervor. Uno que va al mercado o viaja solo, puede fácilmente rezar con atención. Otro que está en su bodega, ocupado en coser los pellejos de vino, está libre para levantar su ánimo al Maestro. El servidor, si no puede llegarse a la Iglesia, porque ha ido de compras al mercado o está en otras ocupaciones, o en la cocina, puede siempre rezar con atención y con ardor. Ningún lugar es indecoroso para Dios. (Homilía sobre la profetisa Ana, 4, 6)

El Papa Gregorio Magno $(\dagger 604)$ enfatiza que el cristiano debe trabajar teniendo como horizonte siempre la eternidad, nuestra patria celestial (cf. Flp 3,20). Al respecto afirma: "Todo cuanto hacemos en este mundo apenas tiene duración hasta la muerte; y llegando esta, arranca el fruto de nuestro trabajo. Pero, cuando trabajamos de cara a la vida eterna, el fruto de nuestro trabajo permanece". (Homilía 27 sobre los evangelios)

Un autor que si bien es cierto ya no está en la época patrística pero conviene citarlo es San Beda $(\uparrow 735)$ el venerable, enseña que el trabajo ofrecido es también oración. Señala: "La oración no consiste solo en las palabras con las que invocamos la clemencia divina, sino también en todo lo que hacemos en obsequio de nuestro creador movidos por la fe". (Comentario al Evangelio de San Marcos)

\section{El trabajo en el Magisterio de la Iglesia}

A partir del Concilio Vaticano II se ha tomado más conciencia del trabajo como materia de santificación del cristiano, en especial de los laicos. Por ejemplo, en ese Concilio se remarca que el trabajo es el ámbito propio donde los laicos se santifican. Leemos en la Lumen gentium:

A los laicos corresponde, por propia vocación, tratar de obtener el reino de Dios gestionando los asuntos temporales y ordenándolos según Dios. Viven en el siglo, es decir, en todos y cada uno de los deberes y ocupaciones del mundo, y en las condiciones ordinarias de la vida familiar y social, con las que su existencia está como entretejida. Allí están llamados por Dios, para que, desempeñando su propia profesión guiados por el espíritu evangélico, contribuyan a la santificación del mundo como desde dentro, a modo de fermento. (Constitución Lumen gentium, $\mathrm{N} .{ }^{\circ} 31$ )

En otro documento del Concilio Vaticano II llamado Gaudium et spes hay un capítulo denominado La actividad humana en el mundo. Ahí se nos indica el gran valor que tiene todo lo que el hombre realiza, cuando ejerce correctamente su inteligencia en el ambiente donde se encuentra. El hombre trabajando se convierte en colaborador de la obra creadora de Dios.

Porque los hombres y mujeres que, mientras procuran el sustento para sí y su familia, realizan su trabajo de forma que resulte provechoso y en servicio de la sociedad, con razón pueden pensar que con su trabajo desarrollan la obra del Creador, sirven al bien de sus hermanos y contribuyen de modo personal a que se cumplan los designios de Dios en la historia. (N. ${ }^{\circ} 34$ )

Asimismo, se enseña que toda actividad humana debe estar ordenada al bien de la persona humana.

Esta es la norma de la actividad humana: que, de acuerdo con los designios $y$ voluntad divinos, sea conforme al auténtico bien del género humano y permita al hombre, como individuo $y$ como miembro de la sociedad, cultivar y realizar íntegramente su plena vocación. (CONCILIO VATICANO II, Constitución Gaudium et spes, N. ${ }^{\circ}$ 35)

Un documento clave para entender el pensamiento de la Iglesia sobre el trabajo es la Encíclica Laborem exercens. En este documento, el Papa Juan Pablo II realizó una magnífica exposición sobre la naturaleza del trabajo y sus implicancias en la vida del hombre. El Santo Padre habló del sentido 
objetivo y subjetivo del trabajo. El sentido objetivo tiene que ver con lo que el hombre realiza: lo que hace. Es decir se trata de lo exterior al hombre. En cambio, el sentido subjetivo se refiere al sujeto del trabajo que es el hombre mismo.

A este respecto, San Juan Pablo II enseñó:

Como persona, el hombre es pues sujeto del trabajo. Como persona él trabaja, realiza varias acciones pertenecientes al proceso del trabajo; éstas, independientemente de su contenido objetivo, han de servir todas ellas a la realización de su humanidad, al perfeccionamiento de esa vocación de persona, que tiene en virtud de su misma humanidad. (Encíclica Laborem exercens, N. ${ }^{\circ}$ 6)

Siendo el sentido objetivo importante, sin embargo, remarca Juan Pablo II, que "en fin de cuentas, la finalidad del trabajo, de cualquier trabajo realizado por el hombre - aunque fuera el trabajo «más corriente», más monótono en la escala del modo común de valorar, e incluso el que más margina- permanece siempre el hombre mismo". (Encíclica Laborem exercens, n. 6) Es la dimensión subjetiva la que debemos de cuidar para que el trabajo respete siempre la dignidad de la persona humana.

Quisiera señalar asimismo lo indicado por el Papa Benedicto XVI en la Encíclica Caritas in veritate. El hoy Papa emérito, afirmó la necesidad de forjar condiciones para un trabajo digno. ¿A qué llamamos un trabajo digno?

Significa un trabajo que, en cualquier sociedad, sea expresión de la dignidad esencial de todo hombre o mujer: un trabajo libremente elegido, que asocie efectivamente a los trabajadores, hombres y mujeres, al desarrollo de su comunidad; un trabajo que, de este modo, haga que los trabajadores sean respetados, evitando toda discriminación; un trabajo que permita satisfacer las necesidades de las familias y escolarizar a los hijos sin que se vean obligados a trabajar; un trabajo que consienta a los trabajadores organizarse libremente y hacer oír su voz; un trabajo que deje espacio para reencontrarse adecuadamente con las propias raíces en el ámbito personal, familiar y espiritual; un trabajo que asegure una condición digna a los trabajadores que llegan a la jubilación. (N. ${ }^{\circ}$ 63.)

\section{Síntesis}

En esta primera parte podemos fijar los siguiente puntos: (1) El trabajo es una actividad que se enraíza en la misma naturaleza del hombre, creado a imagen y semejanza de Dios. (2) El trabajo no es un castigo por el pecado. Lo que trae el pecado es la fatiga en el trabajo. (3) Cristo es el modelo del trabajador. (4) Todo cristiano, inserto en Cristo, puede convertir su trabajo en una ofrenda agradable a Dios. (5) La Iglesia desde sus inicios ha valorado el trabajo honesto, ha defendido los derechos del trabajador; y lo percibe como un medio de santificación.

\section{LA FAMILIA Y EL TRABAJO}

En esta parte vamos a señalar algunas ideas sobre la relación entre la familia y el trabajo. Una primera idea la encontramos en la Encíclica Laborem exercens:

El trabajo es el fundamento sobre el que se forma la vida familiar, la cual es un derecho natural y una vocación del hombre. Estos dos ámbitos de valores - uno relacionado con el trabajo y otro consecuente con el carácter familiar de la vida humana- deben unirse entre sí correctamente y correctamente compenetrarse. El trabajo es, en un cierto sentido, una condición para hacer posible la fundación de una familia, ya que ésta exige los medios de subsistencia, que el hombre adquiere normalmente mediante el trabajo. (S.S. JUAN PABLO II, Encíclica Laborem exercens, N. ${ }^{\circ} 10$ )

\section{El trabajo dignifica la familia}

Comencemos por señalar que la familia es una "comunión de personas". Dado que el trabajo 
perfecciona a las personas entonces redunda en el bien de toda la familia. Hay que señalar un aspecto de vital importancia para la familia: se debe de forjar en la sociedad condiciones de trabajo para todos. Dejar a una persona sin trabajo, no solo conlleva a dejarla en la orfandad, sino que además es toda una familia la que termina perjudicándose.

Cuando decimos que el trabajo dignifica a la familia, nos referimos a un plano material $y$ uno espiritual. El plano material tiene que ver con el hecho de que el trabajo hace posible los medios económicos para la subsistencia. Si en una familia nadie trabaja, lo que existe es la miseria, y esto es un grave atentado contra la dignidad de la personas.

Pero, también está el otro plano, el más noble ciertamente, es el plano espiritual. El trabajo dignifica a la familia en tanto y en cuanto se convierte en medio de crecimiento personal y desde la fe se ve como un camino de santificación.

\section{El trabajo involucra a todos en la familia}

El trabajo compete en primer lugar al jefe de familia. Aunque hoy en día lo común es que sean los dos, padre y madre, quienes laboren. El trabajo del jefe de familia es el que por lo general permite la subsistencia del hogar. Ahora bien, es importante remarcar que este trabajo debe repercutir en el bien de todos los miembros de la familia. Ya hemos indicado que el trabajo debe servir para el mantenimiento digno del hogar. Es la cuestión del sueldo digno. Simplemente aquí menciono que sigue siendo tarea pendiente en muchas sociedades incluida la nuestra.

Además, el trabajo debe ser tal que permita reservar un momento de espacio para la vida familiar. Hoy esto es un gran problema, dado que la gran mayoría de los padres de familia tienen varios trabajos para poder sustentar los diversos gastos que surgen en la vida familiar. Reservar espacios de tiempo para los hijos es fundamental para la correcta educación. Actualmente es común hablar de que en varios hogares los hijos son huérfanos con padres vivos por culpa de horarios inhumanos de trabajo.
En cuanto al trabajo de la madre, se debe reconocer el derecho de las mujeres al trabajo, pero al mismo tiempo, afirmaba el Papa Juan Pablo II, la sociedad está llamada a protegerá las amas de casa.

Una justa remuneración por el trabajo de la persona adulta que tiene responsabilidades de familia es la que sea suficiente para fundar y mantener dignamente una familia y asegura su futuro. Tal remuneración puede hacerse bien sea mediante el llamado salario familiar -es decir, un salario único dado al cabeza de familia por su trabajo y que sea suficiente para las necesidades de la familia sin necesidad de hacer asumir a la esposa un trabajo retribuido fuera de casa- bien sea mediante otras medidas sociales, como subsidios familiares o ayudas a la madre que se dedica exclusivamente a la familia, ayudas que deben corresponden a las necesidades efectivas, es decir, al número de personas a su cargo durante todo el tiempo en que no están en condiciones de asumirse dignamente la responsabilidad de la propia vida. (S.S. JUAN PABLO II, Encíclica Laborem exercens, N. $\left.{ }^{\circ} 19\right)$

\section{En la Exhortación Apostólica Familiaris} consortio la idea es la misma:

Si se debe reconocer también a las mujeres, como a los hombres, el derecho de acceder a las diversas funciones públicas, la sociedad debe sin embargo estructurarse de manera tal que las esposas y madres no sean de hecho obligadas a trabajar fuera de casa y que sus familias puedan vivir y prosperar dignamente, aunque ellas se dediquen totalmente a la propia familia. Se debe superar además la mentalidad según la cual el honor de la mujer deriva más del trabajo exterior que de la actividad familiar. Pero esto exige que los hombres estimen y amen verdaderamente a la mujer con todo el respeto de su dignidad personal, y que la sociedad cree $y$ desarrolle las condiciones adecuadas para el trabajo doméstico. (S.S. JUAN 
PABLO II, Exhortación apostólica Familiaris consortio, N ${ }^{\circ}$ 23)

Y, como hemos visto en la primera parte, no basta lo material. Es decir, no basta que el sueldo sirva para saciar las necesidades familiares. Es necesario que los padres de familia que trabajan conviertan sus labores en medios de santificación. Un padre y una madre que hacen sus trabajos con corrección y lo ofrecen por su familia están haciendo de su ocupación un elemento eficaz de santificación que repercute en todo el hogar.

Los hijos también trabajan. ¿En qué sentido? En el hecho de que toda ocupación es trabajo. Por ello, las ayudas domésticas y el estudio de los hijos deben ser considerados también como un trabajo. En este sentido, se les debe inculcar a los hijos desde pequeños: la responsabilidad, el ofrecimiento de obras y el servicio.

El trabajo exige responsabilidad en los hijos. Es importante establecer metas concretas. Por ejemplo, señalarles la cantidad de horas de estudio que deben hacer cada día. Esto implica que los padres establezcan un control sobre sus hijos. Una cuestión que nos lleva otra vez a señalar el problema actual de la ausencia de los padres en el hogar por un trabajo demasiado absorbente. Además, junto con el estudio, los padres tienen la obligación de enseñar a sus hijos a colaborar en los trabajos domésticos. Formar a los hijos en el trabajo implica hacerles tomar conciencia que deben contribuir en el hogar con tareas sencillas pero significativas, como el orden de las cosas, la limpieza de los utensilios, el aseo de sus cuartos, etc.

Los padres de familia están llamados a educar a sus hijos en la santificación de sus ocupaciones. Esto forma parte de esa educación en la fe propia de los padres, que como sabemos, son los primeros evangelizadores de los hijos. Cuando se les enseña a los hijos a ofrecer las horas de estudio, se les abre un panorama trascendente a esa ocupación. Permítanme en este momento señalar lo dicho por Don Bosco, quien pedía a sus jóvenes que invocaran a San José, pues es el patrono de los trabajadores: "No olvidéis que es el protector de todos los trabajadores y que también de los jóvenes que estudian, porque el estudio es trabajo". (Charlas)

\section{La Eucaristía y la vida del trabajo familiar}

La Eucaristía es el centro de la vida cristiana. Toda familia católica debe considerar la Eucaristía como la fuente de donde mana la gracia de Cristo, que permite vivir como una comunidad de vida y amor. Además, el mismo trabajo familiar debe ser insertado en la celebración eucarística. Cada domingo -que es el Día del Señor-, la familia está llamada a llevar al altar los afanes y esfuerzos que demanda el trabajo de cada uno de sus miembros. En otras palabras, cuando la familia cristiana se reúne en torno al altar del Señor para celebrar la Eucaristía debe ofrecer las labores de cada día. Participar en la Eucaristía, que es la actualización del sacrificio de Cristo al Padre con la fuerza vivificadora del Espíritu Santo, compromete a la familia a hacer de su trabajo una ofrenda grata a Dios. "Ya desde la reunión litúrgica, el Sacramento de la Eucaristía nos compromete en la realidad cotidiana para que todo se haga para gloria de Dios". (BENEDICTO XVI, Exhortación apostólica Sacramentum caritatis, N. ${ }^{\circ}$ 79)

\section{Síntesis}

Como ideas que sintetizan esta segunda parte podemos señalar: (1) El trabajo permite que cada miembro de la familia se perfeccione. (2) En una familia, todos están llamados a trabajar, cada uno según su propio estado. En este sentido, los hijos trabajan en cuanto realizan con responsabilidad sus deberes propios ya sea como hijos o como estudiantes. (3) Desde la fe, el trabajo debe ser percibido como un medio privilegiado para la santificación de la familia. Es una forma como crece la familia en su trato con Dios. (4) La Eucaristía dominical es el centro del cual brota la gracia para que cada miembro de la familia haga de su trabajo una ofrenda agradable a Dios por medio de Jesucristo.

Por último, es necesario remarcar que una cuestión abierta al diálogo y a reflexiones que bien ameritarían otro artículo es cómo 
armonizar en la realidad el trabajo de los padres de familia, tan necesario no solo para el bienestar material sino también para el propio perfeccionamiento de la persona, y la permanencia en el hogar. No se trata aquí de establecer dogmas, sino principios iluminadores que hemos desplegado a lo largo del artículo. 


\section{REFERENCIAS}

BENEDICTO XVI, Exhortación apostólica Sacramentum caritatis.

CONCILIO VATICANO II, Constitución Lumen gentium.

CONCILIO VATICANO II, Constitución Gaudium et spes.

S.S. JUAN PABLO II, Encíclica Laborem exercens.

S.S. JUAN PABLO II, Exhortación apostólica Familiaris consortio.

S.S. BENEDICTO XVI, Encíclica Caritas in veritate.

Fecha de recepción: 12 de febrero 2015

Fecha de aceptación: 08 de abril 2015 